
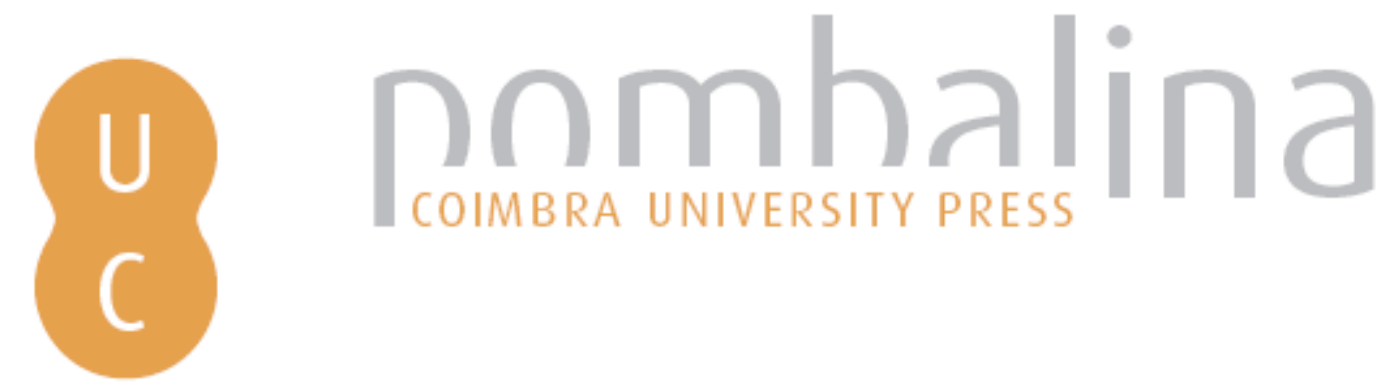

\title{
Uma revisão da modelagem fuzzy da interface continente e oceano para identificar locais vulneráveis à erosão
}

\author{
Autor(es): $\quad$ Silva, L. M.; Gonçalves, R. M. \\ Publicado por: Imprensa da Universidade de Coimbra \\ URL \\ persistente: URI:http://hdl.handle.net/10316.2/31438 \\ DOI: $\quad$ DOI:http://dx.doi.org/10.14195/978-989-26-0531-9_15 \\ Accessed : $\quad$ 26-Apr-2023 03:32:59
}

A navegação consulta e descarregamento dos títulos inseridos nas Bibliotecas Digitais UC Digitalis, UC Pombalina e UC Impactum, pressupõem a aceitação plena e sem reservas dos Termos e Condições de Uso destas Bibliotecas Digitais, disponíveis em https://digitalis.uc.pt/pt-pt/termos.

Conforme exposto nos referidos Termos e Condições de Uso, o descarregamento de títulos de acesso restrito requer uma licença válida de autorização devendo o utilizador aceder ao(s) documento(s) a partir de um endereço de IP da instituição detentora da supramencionada licença.

Ao utilizador é apenas permitido o descarregamento para uso pessoal, pelo que o emprego do(s) título(s) descarregado(s) para outro fim, designadamente comercial, carece de autorização do respetivo autor ou editor da obra.

Na medida em que todas as obras da UC Digitalis se encontram protegidas pelo Código do Direito de Autor e Direitos Conexos e demais legislação aplicável, toda a cópia, parcial ou total, deste documento, nos casos em que é legalmente admitida, deverá conter ou fazer-se acompanhar por este aviso.

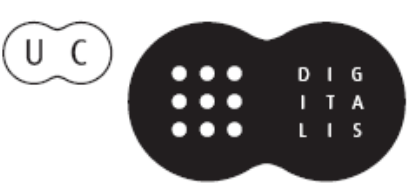





\title{
UMA REVISÁO DA MODELAGEM FUZZY DA INTERFACE CONTINENTE E OCEANO PARA IDENTIFICAR LOCAIS VULNERÁVEIS À EROSÁO
}

\author{
A REVIEW OF FUZZY MODELING OF INTERFACE CONTINENT \\ AND OCEAN TO IDENTIFY VULNERABLE SITES TO EROSION
}

Resumo - O avanço da urbanização vem provocando grandes transformações nas relaçôes sociais e morfológica da área costeira. As informações cartográficas e históricas quando comparadas caracterizam a configuração espacial de um ambiente em estudo, tornando-se uma ferramenta poderosa para detectar mudanças espaciais de determinadas feiçóes. O presente trabalho tem como objetivo revisar a modelagem fuzzy (nebulosa) aplicada à classificação da vulnerabilidade costeira. Os resultados esperados são a aplicação da modelagem através da classificação temática da área de estudo. O método a ser aplicado utiliza variáveis linguísticas que caracterizam a abordagem fuzzy, ideal para estudos onde existem incertezas capazes de serem modeladas matematicamente gerando como produto uma classificação local.

Palavras chaves - Erosão Costeira. Vulnerabilidade. Interface Oceano Continente. Lógica Fuzzy.

Abstract - The urbanization advance in coastal areas has been changing social relations and morphological aspects. The cartographic and historical information when it is compared over time characterize the spatial configuration of an environment under study, and become a powerful tool to detect spatial changes of cartographic features. This study aims to review the fuzzy classification modeling applied to coastal vulnerability. The expected results are the model implementation through the classification coverage classes of the case study. The applied method uses linguistic variables, which characterize the fuzzy

\footnotetext{
${ }^{1}$ Universidade Federal de Pernambuco - UFPE. Centro de Tecnologia e Geociências - CTG. Departamento de Engenharia Cartográfica, Recife - PE. Programa de Pós-Graduação em Ciências Geodésicas e Tecnologias da Geoinformação; luciana_maria15@hotmail.com; rodrigo.mikosz@ufpe.br
} 
approach, ideal for uncertainties studies able to be mathematically modelled, generating a local classification as a product.

Keywords - Coastal Erosion. Vulnerability. Interface Ocean Continent. Fuzzy Logic.

\section{1 - Introduçáo}

As praias são definidas em ALMEIDA (2008) como feiçóes geológicas temporárias e movimentadas, estando entre os sistemas físicos da superfície da terra com maior dinâmica.

O ambiente praial pode ser dividido em três partes: pós-praia, praia (estirâncio) e ante praia. Em um estudo realizado por ARAÚJO et al. (2007) foi possível analisar a ocupação urbana das praias de Pernambuco, onde no setor Metropolitano do Estado 47\% de sua extensão indicava a ocupação concomitante da pós-praia e da praia.

Nos últimos 60 anos, a praia de Boa Viagem e Pina localizada no município de Recife-PE, Brasil vem sendo urbanizada próximo à linha de costa. Diante das construçóes cada vez mais sobre a pós-praia e até mesmo na praia (estirâncio), observam-se problemas de erosão costeira e de vulnerabilidade. Com aproximadamente 8,5 km de extensão a linha de costa desta área vem sendo modificada por processos antrópicos. Segundo SMITH (1991), praias urbanas são aquelas que circundam cidades, bairros ou balneários consolidados de diversos níveis de desenvolvimento. Ou seja, são as praias sob ocupação e influência humana direta, e utilizada, tanto pelas populaçôes locais como por visitantes.

A erosão praial é um problema sério que ocorre em vários lugares do mundo podendo alcançar estágios bastante elevados, assim como ocorre ou ocorreu em outras praias no mundo e no Brasil, por exemplo, na Espanha, em trechos do litoral, a urbanização não deixou espaço suficiente para dinâmica costeira ocasionando prejuízos ambientais, comerciais e recreativos, (DOODY, 2001). No estado de Delaware, localizado na costa leste dos Estados Unidos o enorme valor das propriedades ao longo da costa e o valor econômico da indústria do turismo costeiro combinam para criar um problema de gestão de recursos naturais que é particularmente difícil de resolver, pois na medida em que a indústria do turismo nessa área se expande, há o aumento de barreiras para evitar o avanço do mar devido à erosão das praias, (DANIEL, 2001).

A vulnerabilidade costeira à erosão consiste em diversos processos morfodinâmicos. Diversos estudos vêm sendo realizados para analisar os níveis da vulnerabilidade, em geral utilizando parâmetros geomorfológicos, taxa de variação, variaçôes do nível do mar, altura de ondas, fatores antrópicos, atributos naturais etc., como pode ser visto em MAZZER et al. (2008), MALLMANN (2008), MALLMANN e ARAÚJO (2010) e RAPOSEIRO e FERREIRA (2010).

\section{2 - Área de estudo}

O litoral de Recife é formado pelas praias de Pina (1 km de extensão litorânea) e Boa Viagem $(7,38 \mathrm{~km}$ de extensão litorânea) que totalizam aproximadamente $8,5 \mathrm{~km}$ de linha de costa. 
A figura 1 apresenta um mapa de localização do Estado de Pernambuco na Região Nordeste, no Brasil, a ampliação do Estado e em particular um polígono que define os munícipios de Recife e um poligono de Boa Viagem.

Recife, conforme dados do censo de 2010, conta com uma populaçáo de 1.537 .704 habitantes e apresenta uma superfície territorial de $218.498 \mathrm{~km}^{2}$. Limitando-se ao norte com as cidades de Olinda e Paulista, ao sul com o município de Jaboatão dos Guararapes, a oeste com São Lourenço da Mata e Camaragibe, e a leste com o Oceano Atlântico. Ver Tabela 1, com os dados de área e população em 2010 dos municípios na malha urbana de Recife totalizando aproximadamente três milhóes de habitantes.

Segundo MANSO et al, (2006), o litoral pernambucano foi delimitado e setorizado com base na sua situação geográfica e política em 21 municípios, distribuídos numa faixa de $187 \mathrm{~km}$. A costa pernambucana apresenta baixa altitude, chegando a atingir, em vários pontos, cotas inferiores ao nível médio do mar.

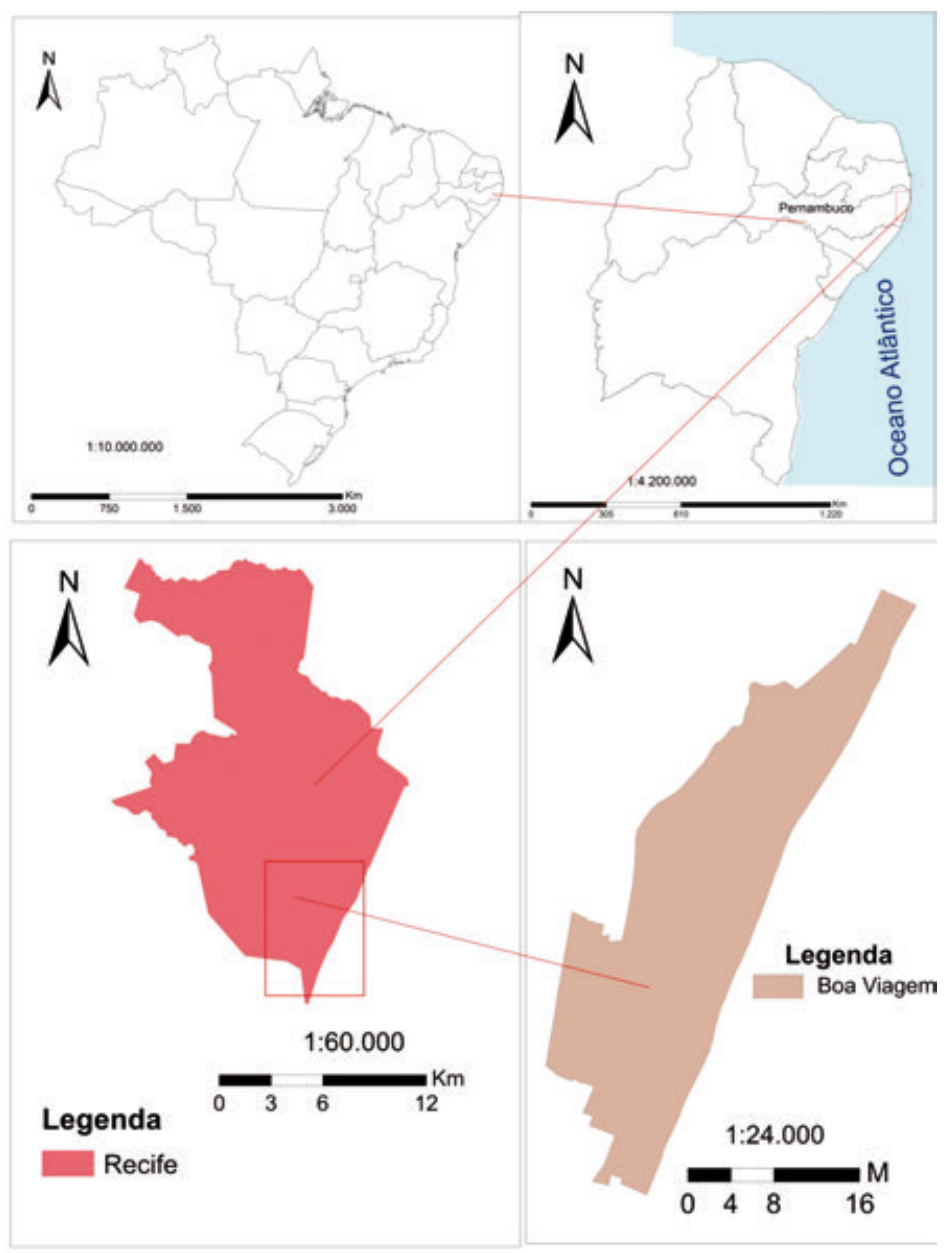

Fig. 1 - Mapa de Localização de Boa Viagem. 
Tabela 1 - Área total e população dos municípios na malha urbana.

\begin{tabular}{lll}
\hline Municípios & Área $(\mathrm{km} 2)$ & Populaçáo (2010) \\
\hline Camaragibe & 51.194 & 144.466 \\
Jaboatấo dos Guararapes & 258.566 & 644.620 \\
Olinda & 41.659 & 377.779 \\
Paulista & 97.364 & 300.466 \\
Recife & 218.498 & 1.537 .704 \\
São Lourenço da Mata & 262.157 & 102.895 \\
Total & 929.438 & 3.107 .930 \\
\hline
\end{tabular}

Fonte: IBGE (2010)

A Praia de Boa Viagem é oceânica e se situa na zona sul da cidade faz parte de um conjunto (composto por um cordão arenoso de orientação aproximada Norte-Sul) de ecossistemas costeiros que se estendem outros $7 \mathrm{~km}$ para o sul, ao longo da praia de Piedade (Município de Jaboatão dos Guararapes), mais detalhes em COSTA et al., (2008), banhada pelo Oceano Atlântico.

A maior parte da praia de Boa Viagem é protegida por cordóes de arenito (beachrocks) paralelos à costa. $\mathrm{Na}$ maré baixa, formam-se várias piscinas naturais ao longo da praia, sendo possível andar sobre os recifes, que são relativamente planos, largos e escorregadios. Quando a maré sobe, os recifes ficam completamente cobertos pela água.

COSTA et al., (2008), relatam que a praia da Boa Viagem e seu segmento mais ao norte, a praia do Pina, chegaram a ser consideradas Unidades de Conservação (58 ha) pela Prefeitura da Cidade do Recife em 2000 e que a construção de uma avenida beira-mar (pistas de rolamento, calçada, mureta, quiosques, banheiros e infraestrutura de lazer) teve forte influência na impermeabilização do terreno e imobilização das dunas, podendo ter levado a um sério deslocamento do frágil balanço sedimentar para o lado da erosão da praia.

O deslocamento do balanço sedimentar também pode ter levado a um aumento da vulnerabilidade da praia a tempestades e ressacas. Como consequências, um enrocamento aderente foi instalado emergencialmente em cerca de $2 \mathrm{~km}$ da praia em 1996. Os fenômenos como ressacas e tempestades apresentam uma tendência recente de aumento de sua frequência e intensidade. Sendo assim a praia poderá estar mais vulnerável a erosão do que a uma mudança (elevação) do nível do mar propriamente dita (COSTA et al., 2008).

\section{3 - Mapeamento da linha de costa}

A zona costeira concentra grande potencial econômico e turístico. Por sua importância a Lei de Gerenciamento Costeiro do Estado de Pernambuco (LGCEP, 2010) que trata dos instrumentos e ocupação da zona costeira em seu capítulo V, artigo 10 inciso 
$2^{\circ}$, propõe que para as áreas urbanizadas não será permitido qualquer tipo de instalaçôes de novas construções, urbanização ou outra forma de utilização do solo na zona costeira, na faixa de $33 \mathrm{~m}$ (trinta e três metros), considerada como "non aedificandi", ou valor superior a este quando comprovado em estudo técnico, medidos perpendicularmente em direção ao continente, a partir da linha de preamar máxima da sizígia atual. Ao longo do litoral de Boa Viagem é possível observar obras muito próximas ao mar demonstrado que a urbanização costeira continua em plena atividade.

Uma forma de realizar eficientemente o gerenciamento é investir no monitoramento geodésico da linha de costa, que desempenha um papel essencial para o planejamento territorial. Vários estudos apresentam o monitoramento da linha de costa como, por exemplo: ROCHA et al. (2008) estudam a aplicação de técnicas de posicionamento GPS (Global Positioning System) tridimensional para localizar a linha de costa, proporcionando a obtenção do Modelo de Elevação Digital (DEM); GONÇALVES (2010) estuda modelos preditivos utilizando dados temporais de linha de costa; entre outros.

Segundo GONÇALVES (2010), o monitoramento da linha de costa se beneficia do posicionamento global (GNSS - Global Navigation Satellite Systems) através de levantamentos geodésicos usando o sistema americano GPS (Global Positioning System); o sistema russo GLONASS (GLObal NAvigation Satellite System); e em breve o sistema europeu Galileo e o sistema chinês Beidou (ou Compass). Outras técnicas que são utilizadas para o mapeamento da linha de costa são as relacionadas ao Sensoriamento Remoto, e entre as mais utilizadas encontram-se: imagens de satélites, fotografias aéreas (aerofotogrametria) e dados (levantamentos), LIDAR (Light Detection and Ranging), que tem um sistema de varredura a laser, aerotransportado ou terrestre, de medição da superfície da terra que tem como principio a emissão e recepção de raios laser, mais detalhes em BOYD e FOODY (2011). Detalhes desses métodos são definidos e discutidos, por exemplo, em BOAK e TURNER (2005).

O uso do sensoriamento remoto torna-se fundamental para obtenção de dados temporais atuando como uma ferramenta poderosa para identificação dos problemas causados pelo não planejamento adequado do espaço físico destinado para urbanização, (LI et al., 2011), além de realizar análises de padrôes, tendências e interações sobre as áreas costeiras.

Segundo PRADO e GALO $(2009,2010)$ técnicas tradicionais de classificação de imagens multiespectrais têm sido a ferramenta usual para o mapeamento temático da cobertura da terra. Tais técnicas foram projetadas para tratar fenômenos que apresentam limites bem definidos e que podem ser facilmente discretizados. Contudo, existem regióes de incertezas e imprecisôes a serem mapeadas, as quais demandam técnicas alternativas, como classificaçóes baseadas em abordagem fuzzy que tem se mostrado mais adequada que as abordagens convencionais. As classificaçôes fuzzy fornecem informações relacionadas com a incerteza na atribuição dos pixels às classes, que podem ser úteis e tornar os mapas temáticos gerados mais confiáveis.

O cenário das áreas costeiras no passado era composto apenas por vegetação e oceano, contendo poucas feiçóes identificáveis para serem utilizadas como controle fotogramétrico. Porém, destaca-se que a qualidade métrica obtida por fotografias aéreas é importante e, muitas vezes, são os únicos dados disponíveis para formar um conjunto de informações temporais em zonas costeiras, (GONÇALVES, 2010). 


\subsection{Vulnerabilidade}

Segundo MAZZER et al. (2008), a vulnerabilidade costeira à erosão está associada a diversos processos morfodinâmicos. Uma ampla gama de métodos é utilizada para analisar os seus níveis, em geral utilizando parâmetros geomorfológicos, taxas de variação da linha de costa, variaçóes do nível do mar, altura de ondas etc.

Atualmente através de dados qualitativos e quantitativos vem se desenvolvendo novos métodos para análise da vulnerabilidade costeira. Aos dados são atribuídos pesos proporcionais à influência da vulnerabilidade sendo utilizado como ferramenta de análise e compartimentaçâo da linha de costa em células costeiras e modelagem espacial em sistema de informação geográfica, tendo como resultado mapas temáticos temporais da vulnerabilidade ao longo da linha de costa, como pode ser visto em MAZZER et al. (2008), MALLMANN e ARAÚJO (2010) e RAPOSEIRO e FERREIRA (2010).

MAZZER et al. (2008), para produzir respostas em relação a vulnerabilidade baseou-se em dois principais pressupostos: as células costeiras são unidades homogêneas de balanço sedimentar e os processos costeiros que atuam na linha de costa em diversas escalas temporais. MALLMANN e ARAÚJO (2010) utilizaram dados qualitativos e quantitativos codificados em pesos proporcionais à sua influência sobre um Índice de Vulnerabilidade Global (IVG) calculado a partir de cinco Índices Parciais de Vulnerabilidade (IPVs), relacionados: à morfologia costeira, à presença de atributos naturais, à influência marinha, aos processos costeiros e a fatores antrópicos. RAPOSEIRO e FERREIRA (2010) basearam-se em quatro etapas principais: divisão da área de estudo em subáreas com características semelhantes em termos de defesa costeira, determinação da probabilidade de ocorrência de cotas de inundaçôes marítimas, estabelecimento de fatores qualitativos associados às consequências de ocorrência, a associação das três etapas iniciais para avaliar os riscos de inundação.

\section{4 - Lógica Fuzzy}

A lógica foi "fundada" por Aristóteles, que estabeleceu um conjunto de regras rígidas para que conclusóes pudessem ser aceitas como logicamente válidas. $\mathrm{O}$ emprego da lógica leva a uma linha de raciocínio lógico baseado em premissas e conclusôes. A lógica binária é uma declaração falsa ou verdadeira, não podendo ser ao mesmo tempo parcialmente verdadeira e parcialmente falsa. A lógica fuzzy permite soluçóes aproximadas. Lotfi Asker Zadeh foi o pioneiro na criação da lógica fuzzy que combinou os conceitos da lógica clássica e os conjuntos de Lukasiewicz (graus de pertinência sendo: 0 , 1/2 e 1, mais tarde expandiu um número infinito de valores entre 0 e 1). Os conceitos fundamentais de lógica fuzzy podem ser visto em ZADEH (1965).

Várias pesquisas têm utilizado a lógica fuzzy como ferramenta de estudo em cartografia, por exemplo: ANTUNES et al. (2003) explicitam a classificação de imagens digitais de alta resolução com base na orientação a objeto, considerando descritores de forma e textura através das regras fuzzy, em uma área teste na zona agrícola no município de Nova Esperança-PR, onde se propôs mapear a mata ciliar ao longo do Rio Porecatú; LAZZAROTTO et al. (2005) utilizaram os indicadores e sistema de inferência fuzzy como avaliação da qualidade de base cartográfica; KERSTING e CENTENO (2007) 
fazem a categorização dos objetos por meio de classificador fuzzy, a partir da definição das funçóes de pertinência baseadas nos descritores de forma, textura e relação entre os objetos; XIMENES et al. (2008), verificam a eficácia do modelo da dinâmica do desmatamento na Amazônia através de uma simulação do índice de similaridade fuzzy elevado; FERNANDES et al. (2010) utilizam a lógica fuzzy e os sistemas de informaçóes geográficas como apoio a decisão ao planejamento do uso do espaço, onde elaboraram um mapa de sugestão, onde o conjunto difuso é utilizado para analisar o resultado dos mapas, entre outras pesquisas.

O raciocínio fuzzy é formado por três etapas: a fuzzificação, a inferência e a defuzzificação.

- Fuzzificação: transformam-se as variáveis do problema em valores fuzzy, ou seja, valores numéricos em termos da linguagem natural.

- Inferência: é a etapa mais importante do raciocínio fuzzy, pois é através dela que é feita a tomada da decisão.

- A defuzzificação: transforma-se o resultado fuzzy em um resultado nítido, ou seja, ele transforma o dado nebuloso em dado quantitativo (consiste em retornar os valores, obter um valor numérico dentro da faixa estipulada pela lógica fuzzy).

\section{5 - Metodologia}

Através de dados de Sensoriamento Remoto são organizadas as informaçôes cartográficas temporais resgatadas possibilitando criar um banco de dados geográfico para extrair informaçóes do processo de mudança temporal antrópica, além de informação espacial da morfologia costeira ao longo da praia de Boa Viagem.

Para desenvolver uma modelagem para vulnerabilidade, pode-se escolher uma abordagem semi-quantitativa, na qual todos os atributos, qualitativos ou quantitativos, são codificados em variáveis linguísticas (1, 2 e 3) utilizando lógica fuzzy de acordo com seu grau de influência sobre a vulnerabilidade da costa à erosão. Todos os dados referentes a cada segmento podem ser organizados em matrizes para possibilitar a álgebra dos mapas.

Para este estudo ser desenvolvido pode-se utilizar como materiais:

- Ortofotos no formato Tif'e Imagens orbitais de Satélites (Quickbird e Geoeye) que servem para representar as informaçóes espaciais da morfologia costeira no processo de vetorização

- E o software ArcGIS 9.3 para a interpretação de feiçóes cartográficas e elaboração de mapas temáticos da morfologia costeira.

- O software Matlab : Fuzzy Logic Toolbox será utilizado para fazer a modelagem da vulnerabilidade, onde a entrada fuzzy será baseada no espaço, possibilitando uma análise quantitativa, os dados onde são inseridos através da Fuzzy Logic Toolbox.

- Utilizaçáo do software Excel $^{\oplus}$, para colocar os resultados.

A seguir na Tabela 2 são apresentados exemplos de classes possíveis de serem marcadas com as variáveis utilizadas, seus intervalos e variáveis linguísticas utilizadas que servem de dados de entrada para a modelagem fuzzy. Após a fuzzificação ocorrerá 
à inferência onde é feito o cálculo dos índices, com seus intervalos e variáveis correspondentes; os dados nebulosos obtidos serão transformados em quantidades numéricas, quantidades estas que servirão para análise dos dados.

Tabela 2 - Classes utilizadas como dados de entrada e suas variáveis linguísticas correspondentes para utilização da Lógica Fuzzy.

\begin{tabular}{cccc}
\hline $\begin{array}{c}\text { Dados Espaciais } \\
(\phi, \lambda, \mathrm{h})\end{array}$ & Classes & \multicolumn{3}{c}{ Variáveis Linguísticas } \\
\hline Características Morfológicas e antrópicas & 1 & 2 & 3 \\
Enrocamento & Presente & Adjacente & Ausente \\
Benfeitorias de Lazer & Presente & Adjacente & Ausente \\
Praia (estirâncio) & Ampla & Média & Estreita \\
Vegetação (dunas frontais e coqueiros) & Alta & Média & Baixa \\
Ante praia & Ampla & Média & Estreita \\
Linha de costa & Estável & Moderada & Instável \\
Urbanizaçấo (tipos de Construçôes) & Alta & Média & Baixa \\
Erosáo costeira & Ausente & Adjacente & Presente \\
\hline
\end{tabular}

\section{6 - Consideraçôes finais}

A praia é um dos grandes pontos recreativos na cidade do Recife, mas encontra-se comprometida por conta da falta de espaço de areia para permanência quando a maré está alta. Através de uma análise temporal, considerando como referência a evolução do espaço onde é possível quantificar as classes selecionadas, obtém-se a modelagem da interface oceano continente.

O enrocamento, proteção feita com rochas, tem a missão de retirar a energia das ondas e proteger a área urbanizada gerando um grande problema que é mútuo, pois prejudica a dinâmica da praia e da maré. A contenção do mar, por conta do mau planejamento da cidade, se torna um problema, pois exige cuidados constantes.

Através da metodologia utilizada para identificar as mudanças ao longo do ambiente praial, destaca-se que a fonte de informação cartográfica servirá como ferramenta fundamental para as análises de informaçóes espaciais e da modelagem, mas ainda existem muitas questóes que precisam ser analisadas e respondidas no que se refere ao diagnóstico e temas ligados ao crescimento da urbanização costeira.

Agradecimentos - Os autores desejam agradecer o apoio financeiro da FACEPE/ CNPq através do projeto de pesquisa PPP/APQ-1242-1.07/10 PPP e ao Laboratório de Cartografia Costeira - LACCOST pela disponibilização de equipamentos e espaço físico para pesquisa. Luciana, à bolsa de mestrado contemplada pela Capes. 


\section{Referências Bibliográficas}

ALMEIDA, H. R. R. C. (2008) - Séries temporais de imagens suborbitais e orbitais de alta resolução espacial na avaliação da morfodinâmica praial no município do Cabo de Santo Agostinho -PE. Dissertação de conclusão de curso, (Mestrado em Ciências Geodésicas) UFPE, 124p.

ANTUNES, A. F. B., LINGNAU, C. \& CENTENO, J. A. S. (2003) - Object Oriented Analysis and Semantic Network for high Resolution Image Classification. Boletim de Ciências Geodésicas, vol.9, nº 2, p. 233-242.

ARAÚJO, M. C. B., SOUZA, S. T., CHAGAS, A. C. O., BARBOSA, S. C. T. \& COSTA, M. F. (2007) - Análise da Ocupação Urbana das Praias de Pernambuco, Brasil. Revista da Gestão Costeira Integrada. 7(2), p. 97-104.

BOAK, E. H. \& TURNER, I. L. (2005) - Shoreline Definition and Detection: A Review. Journal of Coastal Research. 21(4), p. 688-703.

BOYD, D. S. \& FOODY, G. M. (2011) - An overview of recent remote sensing and GIS based research in ecological informatics. Journal of Ecological Informatics. 6, p. 25-36.

COSTA, M. F., ARAÚJO, M. C. B., CAVALCANTI, J. S. S. \& SOUZA, S. T. (2008) - Verticalização da Praia da Boa Viagem (Recife, Pernambuco) e suas Consequências Socioambientais. Revista da Gestão Costeira Integrada. 8(2), p. 233-245.

DANIEL, H. (2001) - Replenishment versus retreat: the cost of maintaining Delaware's beaches. Ocean \& Coastal Management. 44, p. 87-104.

DOODY, J. P. (2001) - Shoreline management - conservation, managementor restoration? National Coastal Consultants. p. 407-419.

FERNANDES, E., BASTOS, L. C. \& MELlO, R. (2010) - Apoio à decisão ao Planejamento do uso do espaço com a utilização de Sistemas de Informaçōes Geográficas e Lógica Difusa. Revista Brasileira de Cartografia, no 62, p. 43-54.

GONÇALVES, R. M. (2010) - Modelagem de tendência a curto-prazo da linha de costa através de dados geodésicos temporais utilizando regressão linear, estimativa robusta e redes neurais artificiais. Tese de conclusâo de curso (Doutorado em Ciências Geodésicas), UFPR, 152p.

IBGE (2010) - Mapa de distribuição da população 2010. Instituto Brasileiro de Geografia e Estatística. Disponível em Internet: http://www.ibge.gov.br/home/geociencias/geografia/mapas_doc1.shtm acessado em 13/07/2011.

KERSTING, A. P. B. \& CENTENO, J. A. S. (2007) - Object Oriented Transmission Line Corridors Classification using LIDAR Technology and a Non-Metric Digital Camera. Boletim de Ciências Geodésicas, vol. $13, \mathrm{n}^{\circ} 2$, p. 295-315.

LAZZAROTTO, D. R., SLUTER, C. R. \& DALMOLIN, Q. (2005) - Avaliação da qualidade de base Cartográfica por meio de indicadores e Sistema de Inferência Fuzzy. Boletim de Ciências Geodésicas: Resumos, vol.11, no 2, p. 297-299.

LGCEP (2010) - Lei de Gerenciamento Costeiro do Estado de Pernambuco. Lei No 14. 258, de 23 de dezembro de 2010 .

LI, C., YIN, J. \& LIU, L. (2011) - Research Overview on Urban Land Use Change Based on Remote Sensing Images. International Journal of Environmental Science and Development, vol. 2, no 1, p. 45-48.

MALLMANN, D. L. B. (2008) - Vulnerabilidade do Litoral Sul de Pernambuco à erosão. Dissertação de conclusão de curso (Mestrado em Oceanografia), UFPE, 152 p.

MALlmanN, D. L. B. \& ARAÚJO, T. C. M. (2010) - Vulnerabilidade do Litoral Sul de Pernambuco à erosão. Tropical Oceanography, vol. 38, no 2, p. 129-151.

MANSO, V. A. V., COUTINHO, P. N., GUERRA, N. C. \& JUNIOR, C. F. A. S. (2006) - Erosão e Progradação do Litoral Brasileiro: Pernambuco. Laboratório de Geologia e Geofísica Marinha - LGGM. Recife: Editorial da Universidade. 
MAZZER, A. M., DillenburG, S. R. \& SOUZA, C. R. G. (2008) - Proposta de método para análise de vulnerabilidade à erosão costeira no sudeste da ilha de Santa Catarina, Brasil, 38(2), p.278-294.

PRADO, F. A. \& GALO, M. L. B. T. (2009) - Classificação de Imagens Multiespectrais Baseada em Abordagem Fuzzy. Revista Brasileira de Cartografia. no 61/03, p.233-244.

PRADO, F. A. \& GALO, M. L. B. T. (2010) - Desenvolvimento de um Sistema Hierárquico de Classificação com Aplicação Baseada em Abordagem Fuzzy. Boletim de Ciências Geodésicas - Curitiba-PR, vol. 16, nº 2, p.309-331.

RAPOSEIRO, P. D. \& FERREIRA, J. C. R. (2010) - A análise da vulnerabilidade e do risco de inundação como ferramenta de apoio à gestão dos territórios litorais sob pressão urbana. Pluris: Planejamento urbano regional integrado e sustentável, p. 1-12.

ROCHA, C. P., ARAÚJO, T. C. M. \& MENDONÇA, F. J. B. (2008) - Aplicação de técnicas de posicionamento GPS tridimensional para localizar linhas de costa: estudo de caso na praia de Boa Viagem, Recife-PE, Brasil. Revista da Gestão Costeira Integrada. 8(2), p. 127-137.

SMITH, R. A. (1991) - Beach resorts: A model of development evolution. Landscape and Urban Planning, 21, p. $189-210$.

XIMENES, A. C., ALMEIDA, C. M. \& AMARAL, S., ESCADA, M. I. S., AGUIAR, A. P. D. (2008) - Modelagem Dinâmica do desmatamento na Amazônia. Boletim de Ciências Geodésicas, vol. 14, no 3, p. 370-391.

ZADEH, L. A. (1965) - Fuzzy Sets, Inform. and Control. 8, p. 338-353. 